\title{
Children infected with meningitis before 1 year of age were at increased risk of disability at 5 years of age
}

\author{
Bedford H, de Louvois J, Halket S, et al. Meningitis in infancy in England and Wales: follow up at age 5 years. BMJ 2001 \\ Sep 8;323:533-6.

\section{QUESTION: In children who had meningitis during their first year of life, what are the sequelae at 5 years of age?}

\section{Design}

Inception cohort of children who survived an acute attack of meningitis in their first year.

\section{Setting}

General practices in the UK.

\section{Patients}

1717 children who contracted meningitis during their first year. Sex and age matched children from the same general practice as the index child formed the control group. Data at 5 years of age were available for 1584 children (92\%) who had had meningitis and 1391 control group children (94\%).

\section{Assessment of prognostic factors}

General practitioners (GPs) completed questionnaires on children, which described problems in health, neuromotor development, learning, vision, hearing, speech and language, behaviour, and presence of seizure disorders. Parents completed questionnaires on their child's health, development, and schooling. Gestational age, birth weight, the organism that caused the meningitis, and age at time of infection were also recorded.

\section{Main outcome measures}

Disability (none to severe) at 5 years of age. Children with no disability had no developmental problems, but might have such conditions as asthma or eczema. Children with mild disability had a condition prevalent in the age group, including middle ear disease, squint, febrile convulsions, or behavioural problems. Children with moderate disability attended mainstream school, with or without help, and had impaired functioning, but no severe intellectual or developmental disability. Conditions in this group included mild neuromotor disabilities, intellectual impairment, moderate sensorineural hearing loss, mild to moderate visual impairment, treatment controlled epilepsy, and uncomplicated hydrocephalus. Children with severe disability were unable to attend mainstream school and conditions included severe neuromotor and intellectual impairment, severe seizure disorders, and severe visual or auditory impairment.

\section{Main results}

32 children (1.9\%) who survived the initial meningitis attack died before 5 years of age. Children who had had meningitis were at greater risk of health and developmental problems than control group children $(\mathrm{p}<0.001$ for all problems). They had increased risk of severe or moderate disability (247 children [15.6\%], $\mathrm{p}<0.001)$ and mild disability (461 children [29\%], $\mathrm{p}<0.001$ ) (table). Children infected with meningitis within the first month of life had more health and developmental problems than those infected after 1 month of age $(p=0.03)$. Severity of disability varied according to type of organism responsible for the infection; the highest rates of severe or moderate disability occurred with meningitis caused by less prevalent organisms.

\section{Conclusion}

Children who had meningitis in their first year of life had a 10-fold increase in the risk of moderate or severe disability at 5 years of age compared with non-infected children.

Sources of funding:

Action Research and the Fayed Charitable Foundation.

For correspondence: $M s$ $H$ Bedford, Centre for Paediatric Epidemiology and Biostatistics, Institute of Child Health, London, UK.

A modified version of this abstract appears Evidence-Based Medicine.

Risk of disability in children who had meningitis in their first year of life Severity of disability at 5 years Meningitis Control Relative risk $(95 \% \mathrm{Cl})$

\begin{tabular}{lccc} 
Severe or moderate & $16 \%$ & $1.5 \%$ & $10.33(6.60$ to 16.0$)$ \\
\hline Mild & $29 \%$ & $20 \%$ & $1.47(1.29$ to 1.68$)$ \\
\hline
\end{tabular}

\section{COMMENTARY}

The study by Bedford $e t$ al is important because large, long term follow up studies of children who have had infectious diseases are rare. Outcome measurement in the study is particularly strong. Questionnaire data from both GPs and parents are welcome because this approach increases the validity of the data and provides a more complete picture of the health and development of each child. The researchers achieved a remarkable response rate from both GPs and parents, which adds weight to the external validity of the study. The categories of the scale used to assess disability are based on functional level and, perhaps most helpful, related to educational requirements.

In this study, the authors analysed the data to control for the effects of both gestational age and birth weight on the outcomes. However, caution might be exercised in interpreting the results because the first year of life is a hazardous period, and the influence of other neonatal problems and comorbidities should be considered.

This study is relevant to all nurses working with infants and children because it allows them to give parents accurate information about the likelihood of long term effects on development after meningitis during infancy. One worrying finding is that by 5 years of age, almost $8 \%$ of children in the meningitis group had not had a hearing test, which is recommended, and about $25 \%$ of children in both groups had not had a vision test. Nurses can ensure that all children receive adequate developmental testing, particularly when children are at an increased risk.

For the future, this study suggests cautious optimism about the potential effect of meningitis prevention programmes. Current Haemophilus influenzae type b and Neisseria meningitides group c vaccination programmes would have prevented many of the children in this study from contracting the disease. Furthermore, much effort is being made to reduce neonatal Group B Streptococcus infections by reducing maternal carriage. The use of a Streptococcus pneumoniae vaccine is also being considered.

Edward Purssell, RGN, RSCN, MSc Lecturer in Paediatrics King's College London London, UK 